\title{
DIAPHRAGMLESS SHOCK TUBE FOR PRIMARY DYNAMIC CALIBRATION OF PRESSURE METERS
}

\author{
A. Svete ${ }^{1}$, J. Kutin ${ }^{2}$ \\ ${ }^{1}$ University of Ljubljana, Faculty of Mechanical Engineering, Ljubljana, Slovenia, andrej.svete@fs.uni-lj \\ ${ }^{2}$ University of Ljubljana, Faculty of Mechanical Engineering, Ljubljana, Slovenia, joze.kutin@fs.uni-lj
}

\begin{abstract}
:
In conventional shock tubes with a diaphragm many effects related to the burst of the diaphragm can influence the shock formation and thus prevent an ideal pressure step change predicted by the shock tube measurement model being generated. This paper presents a newly developed diaphragmless shock tube, in which a diaphragm is replaced with a quick-acting pneumatic valve. The developed shock tube has a capability to generate pressure steps calculable from its measurement model with a relative expanded uncertainty of less than 0.025 , which can be used as the input signal in primary calibrations of pressure meters.
\end{abstract}

Keywords: time-varying pressure; primary calibration method; diaphragmless shock tube; frequency characteristics

\section{INTRODUCTION}

A shock tube is only known time-varying pressure generator capable of providing SItraceable high-frequency time-varying pressure calibrations [1]. It consists of two straight gas-filled tubes with the same circular cross-section that are initially separated: a high pressure driver section and a lower pressure driven section. An instantaneous opening of the connection between the two sections generates a shock wave that propagates through the driven gas. The reflection of the shock front off the end-wall of the driven section, where the pressure meter to be calibrated is mounted, causes a rapidly rising step change in the pressure at this point. By assuming a calorically perfect gas and an adiabatic flow, the amplitude of the pressure step upon reflection of the initial shock front can be calculated using traceable measurements of the shock wave velocity $W$ and the initial, stationary absolute pressure $p_{1}$ and temperature $T_{1}$ of the gas in the driven section as [2]:

$$
\Delta p=2 p_{1} \frac{\gamma_{1}}{\left(\gamma_{1}^{2}-1\right)}\left(M_{\mathrm{s}}^{2}-1\right)\left(\frac{M_{\mathrm{s}}^{2}\left(3 \gamma_{1}-1\right)+3-\gamma_{1}}{M_{\mathrm{s}}^{2}+\frac{2}{\left(\gamma_{1}-1\right)}}\right),
$$

where $\gamma_{1}$ is the adiabatic index, $M_{\mathrm{s}}=W / a_{1}$ is the shock wave Mach number, $a_{1}=\sqrt{\gamma_{1} R_{1} T_{1}}$ is the speed of sound and $R_{1}$ is the specific gas constant. After the shock wave has been reflected, the pressure remains constant until the arrival of a second shock front, resulting from the initial shock front's partial reflection from the contact surface between the driver and the driven gases. The extremely rapid rise in pressure (of the order of 1 ns [3]) can excite frequencies in the $\mathrm{MHz}$ range with a low frequency limit that is proportional to the reciprocal of the period of time during which the post-shock pressure remains constant.

In the conventional shock tubes the shock waves are generated by bursting the diaphragm, usually induced by increasing pressure in the driven section. The characteristics of the diaphragm material and the way in which it bursts influence the shock formation, which causes large uncertainties associated with the repeatability of the generated pressure step [4]. Another major disadvantage of the diaphragm shock tubes is that the diaphragm has to be replaced after each test, which requires the shock tube to be completely disassembled and reassembled before each test. This influences the reproducibility of the generated pressure steps. In order to improve the calibration and measurement capability of the shock tube method, the shock tube with a quick-acting pneumatic valve has been developed.

\section{DIAPHRAGMLESS SHOCK TUBE}

The diaphragmless shock tube developed for primary dynamic calibrations of pressure meters is shown in Figure 1. The shock tube consists of $1 \mathrm{~m}$ long driver section and $6 \mathrm{~m}$ long driven section, both manufactured from stainless steel tube with a 
$7.5 \mathrm{~mm}$ thickness wall. In order to eliminate the imperfections at the junctions that could generate secondary shock waves, the driver and the driven section of the same inner diameter as the effective flow-section diameter of the quick-acting valve of $40 \mathrm{~mm}$ are connected to the valve and the end-walls with socket weld flanges. The integrated quickacting pneumatic valve (Ista Pneumatics, KB-40-70) allows driver pressures up to $7 \mathrm{MPa}$, which enables generating pressure steps at the end-wall of the driven section of magnitude up to approximately 1.3 $\mathrm{MPa}$ when using nitrogen. The shock tube is rigidly attached with five massive mounting blocks along its length in order to eliminate the vibrations of the tube generated by the opening of the valve and the motion of the shock waves, which could affect the more acceleration-sensitive pressure meters during calibration.

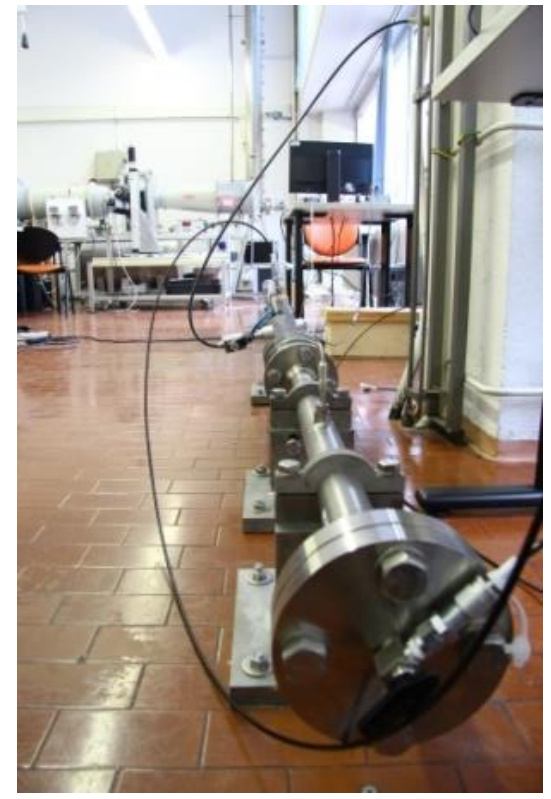

Figure 1: Developed diaphragmless shock tube with a quick-acting valve

In order to obtain the pressure steps with the shock tube measurement model (1), the initial atmospheric driven pressure $p_{1}$ is measured with a barometric pressure transducer (Mensor, CPR6000, measuring range $75 \mathrm{kPa}$ to $115 \mathrm{kPa}$, expanded measurement uncertainty $(k=2) 50 \mathrm{~Pa})$. The initial, stationary driver gauge pressure $p_{4, \mathrm{~g}}$ is measured with a pressure transducer (Mensor, CPR6000, measuring range 0 to $10 \mathrm{MPa}$, expanded measurement uncertainty $(k=2) 0.01 \%$ of reading but not less than $400 \mathrm{~Pa}$ ) connected to the shut-off valve integrated in the end-wall of the driver section shown in Figure 2. The shut-off valve is also used to vent the gas from the tube to the atmosphere. The initial temperature of the gas in driven section $T_{1}$ is inferred from the temperature of the driven section wall measured with Pt100 resistance temperature sensor (TetraTec Instruments, WIT-S) mounted in the middle of the driven section length. The temperature sensor is connected to the digital transmitter (Pico Technology, PT-104, cal. measuring range $18{ }^{\circ} \mathrm{C}$ to $28^{\circ} \mathrm{C}$, expanded measurement uncertainty $(k=2) 0.2^{\circ} \mathrm{C}$, USB PC connection). Measured initial absolute pressure $p_{1}$ and temperature $T_{1}$ of the gas in the driven section are used to obtain its adiabatic index $\gamma_{1}$ and the specific gas constant $R_{1}$ using the NIST REFPROP database.

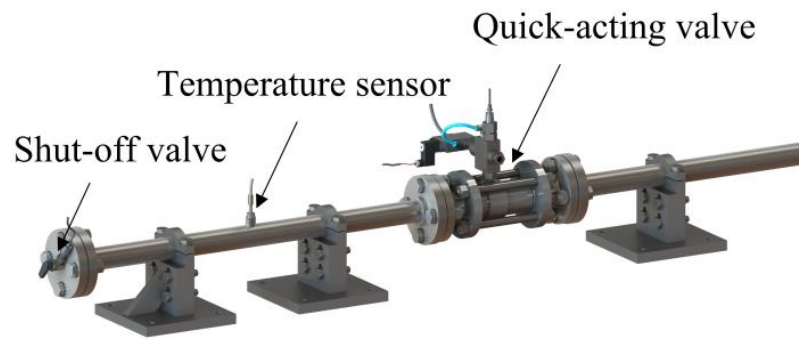

Figure 2: Schematic view of the driver section

The shock wave velocity along the driven section $W$ is determined by measuring time delays between the shock front detections using four identical piezoelectric pressure transducers (Kistler, 603CAA, nominal sensitivity $-50 \mathrm{pC} \mathrm{MPa}^{-1}$, measuring range 0 to $100 \mathrm{MPa}$, natural frequency > $500 \mathrm{kHz}$, acceleration sensitivity $\leq 1.4 \mathrm{~Pa} \mathrm{~m}^{-1} \mathrm{~s}^{2}$ ), which are flush mounted in the side wall of the driven section (see Figure 3) at evenly spaced distances of $1.5 \mathrm{~m}$ from $0.75 \mathrm{~m}$ to $5.25 \mathrm{~m}$ downstream of the quick-acting valve. The output signals of all four pressure transducers are connected in series to the same input of the charge amplifier (Dewetron, DAQP-CHARGE-B, sensitivity $0.05 \mathrm{~V} \mathrm{pC}^{-1}$, full scale output $-5 \mathrm{~V}$ to $5 \mathrm{~V}$, frequency range $0.07 \mathrm{~Hz}$ to $200 \mathrm{kHz}(-3 \mathrm{~dB})$ ).

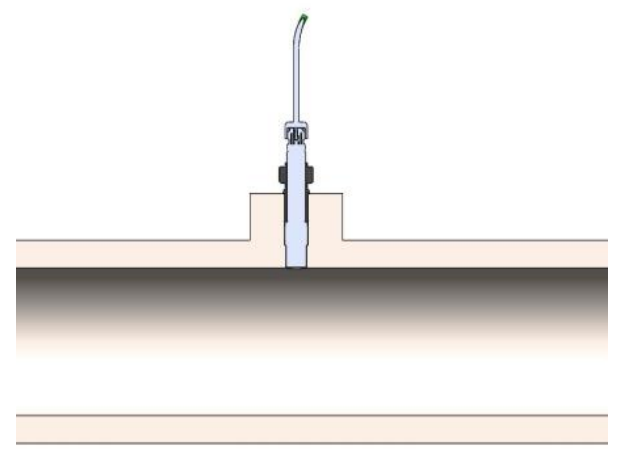

Figure 3: Cross-sectional view of the side wall of the driven section with a flush mounted pressure transducer

The developed shock tube was used to determine frequency characteristics of the piezoelectric pressure measurement system. The piezoelectric pressure measurement system under test consists of pressure transducer (Kistler, 7031, nominal sensitivity $-534 \mathrm{pC} \mathrm{MPa}^{-1}$, measuring range 0 to $25 \mathrm{MPa}$, natural frequency approximately $80 \mathrm{kHz}$, acceleration sensitivity $<1 \mathrm{~Pa} \mathrm{~m}^{-1} \mathrm{~s}^{2}$ ) and charge 
amplifier (Kistler, 5018A, measuring range $2 \mathrm{pC}$ to $2200000 \mathrm{pC}$, full scale output $-10 \mathrm{~V}$ to $10 \mathrm{~V}$, frequency range $0.16 \cdot 10^{-5} \mathrm{~Hz}$ to $200 \mathrm{kHz}(-3 \mathrm{~dB})$ ).

The voltage outputs from the charge amplifiers are acquired by a digitizer (National Instruments, NI 9775 , set input range $-5 \mathrm{~V}$ to $+5 \mathrm{~V}$, resolution 11 bit, set sampling frequency $1 \mathrm{MHz}$, analog filter bandwidth $13.9 \mathrm{MHz} \quad(-3 \mathrm{~dB}))$. The dataacquisition platform (National Instruments, CompactDAQ) also employs a digital output module (National Instruments, NI 9474, output signal $24 \mathrm{~V} \mathrm{DC}$ ) to enable the computer-controlled opening and closing of the quick-acting pneumatic valve using the analog voltage signal supplied to the electro-pneumatic valve. The signal acquisition and processing, and the actuation of the opening and closing of the valve are realized in the LabVIEW programming environment.

\section{DETERMINATION OF THE FREQUENCY CHARACTERISTICS}

Assuming that the pressure measurement system under calibration, consisting of a piezoelectric transducer, a charge amplifier and a digitizer, exhibits linear behavior, its frequency response can be determined as the ratio of the discrete Fourier transforms (DFTs) of the output signal $F_{\text {out }}(\omega)$ and known input signal $F_{\text {in }}(\omega)$. The DFT of a signal on a finite interval implies the approximation of the Fourier series of its infinite periodic extension. Due to the fact that the step signal has unequal values at the interval end points, the periodic extension has discontinuities resulting in significant errors [5]. Therefore, prior to applying the DFT, both the input and output signals are truncated after constant poststep value of the output signal $P$ is reached and then windowed using the Gans-Nahman technique [6]. This technique involves the extension of the original, truncated step signal $p(n \Delta t)$, into the signal $p^{*}(n \Delta t)$ as:

$$
p^{*}(n \Delta t)=\left\{\begin{array}{ll}
p(n \Delta t), & \text { for } n=0, \mathrm{~K}, N-1 \\
P-p(n-N) \Delta t, & \text { for } n=N, \mathrm{~K}, 2 N-1
\end{array},\right.
$$

which satisfies $p^{*}(0)=p^{*}(2 N \Delta t)$, where $N$ is the number of samples of the truncated signal and $\Delta t$ is the sampling period. Although the technique doubles the length of the time signals, the frequency resolution of the original signal is preserved as the DFT solution is zero at the even harmonics. Further, the amplitude-frequency characteristic of the measurement system under test is determined as:

$\beta(\omega)=|G(\omega)|$,

where $G(\omega)=F_{\text {out }}(\omega) / F_{\text {in }}(\omega)$, and phasefrequency characteristic as:

$$
\phi(\omega)=-\operatorname{atan}(\operatorname{Im}(G(\omega)) / \operatorname{Re}(G(\omega)))
$$

where $\omega=k \frac{\pi}{N \Delta t}$ for $k=0,1,3,5 \ldots, N-1$.

\section{RESULTS}

The tests were performed using dry nitrogen (5.0) at the atmospheric initial driven pressure and at the initial driver gauge pressures of $3 \mathrm{MPa}, 5 \mathrm{MPa}$ and $7 \mathrm{MPa}$. Before the tests, the air was evacuated from the shock tube through the open end of the driven section by blowing through the whole shock tube with nitrogen. After closing the end of the driven section, the quick-acting valve and the shut-off valve were held open for a few minutes to provide atmospheric pressure of the nitrogen in the driven section. After closing the quick-acting valve, the driver section was pressurized to the required pressure. Before the actuation of the opening of the quick-acting valve, the initial temperature and absolute pressure (atmospheric pressure) of the gas in the driven section were measured.

Table 1 presents measured initial absolute pressures (atmospheric pressure) $p_{1}$ and temperatures $T_{1}$ of the gas in the driven section, the adiabatic index $\gamma_{1}$, the speed of sound $a_{1}$ and measured end-wall shock wave velocities $W$ for the tests conducted at driver gauge pressures $3 \mathrm{MPa}$, $5 \mathrm{MPa}$ and $7 \mathrm{MPa}$.

Table 1: Measured $p_{1}, T_{1}, \gamma_{1}, a_{1}$ and $W$ at initial driver gauge pressures $p_{4, \mathrm{~g}}$ of $3 \mathrm{MPa}, 5 \mathrm{MPa}$ and $7 \mathrm{MPa}$

\begin{tabular}{|c|c|c|c|c|c|}
\hline $\begin{array}{c}\boldsymbol{p}_{\mathbf{4}, \mathbf{g}} \\
\mathbf{M P a}\end{array}$ & $\begin{array}{c}\boldsymbol{p}_{\mathbf{1}} \\
\mathbf{M P a}\end{array}$ & $\begin{array}{c}\boldsymbol{T}_{\mathbf{1}} \\
\mathbf{K}\end{array}$ & $\boldsymbol{\gamma}_{\mathbf{1}}$ & $\begin{array}{c}\boldsymbol{a}_{\mathbf{1}} \\
\mathbf{m} / \mathbf{s}\end{array}$ & $\boldsymbol{W} \mathbf{~ m / s}$ \\
\hline 3 & 0.09893 & 295.5 & 1.4013 & 351.4 & 612.6 \\
\hline 5 & 0.09891 & 295.1 & 1.4013 & 351.1 & 661.7 \\
\hline 7 & 0.09890 & 295.4 & 1.4013 & 351.3 & 696.1 \\
\hline
\end{tabular}

Using the measurement model of the shock tube (1), the amplitudes of the input pressure signals provided to the transducer under test were determined. A relative expanded uncertainty of the amplitudes of the pressure steps generated within the developed shock tube has been estimated to be less than 0.025 (for the detailed uncertainty analyses see [7]). Figure 4 presents the input signals and the output response signals, which were obtained by considering the sensitivity of the piezoelectric pressure measurement system specified by its manufacturer. The signals are presented in the interval from $-0.1 \mathrm{~ms}$ to $1 \mathrm{~ms}$, where the arrival time of the shock front at the end-wall, which was determined with the help of the measured shock wave velocity along the driven section, is set to $t=0$. 


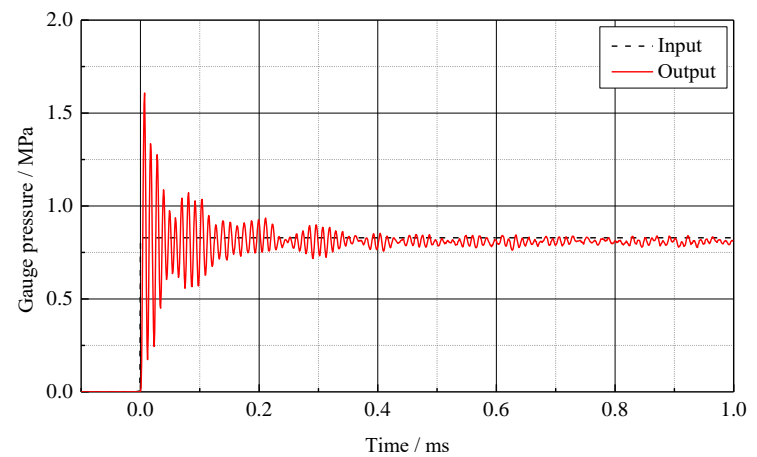

(a)

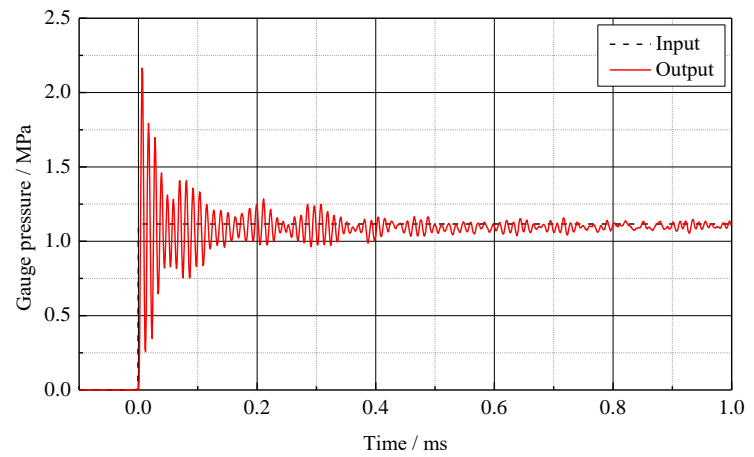

(b)

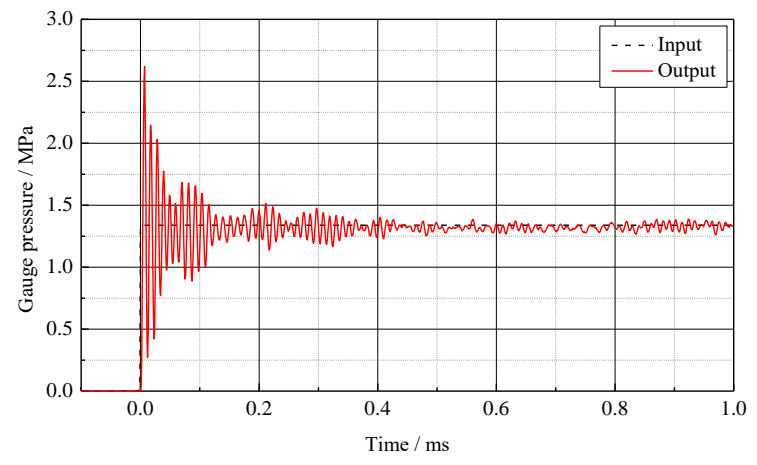

(c)

Figure 4: Pressure steps generated at the end-wall of the driven section at: (a) $p_{4, \mathrm{~g}}=3 \mathrm{MPa}$, (b) $p_{4, \mathrm{~g}}=5 \mathrm{MPa}$, (c) $p_{4, \mathrm{~g}}=7 \mathrm{MPa}$

Figure 5 presents the amplitude-frequency characteristics of the piezoelectric pressure measurement system in the frequency range up to Nyquist frequency of $500 \mathrm{kHz}$. The obtained amplitude-frequency characteristics were normalized with the static measurement sensitivity of the pressure measurement system $\beta(0)$. An average static sensitivity of the pressure measurement system obtained with the calibration using the shock tube differs from that specified by the manufacturer of the pressure measurement system by less than $3 \%$. From the figure it is seen that the amplitude-frequency characteristics of the pressure measurement system exhibit two peaks, one at the frequency of approximately $82.5 \mathrm{kHz}$ and one at the frequency of approximately $92.5 \mathrm{kHz}$.

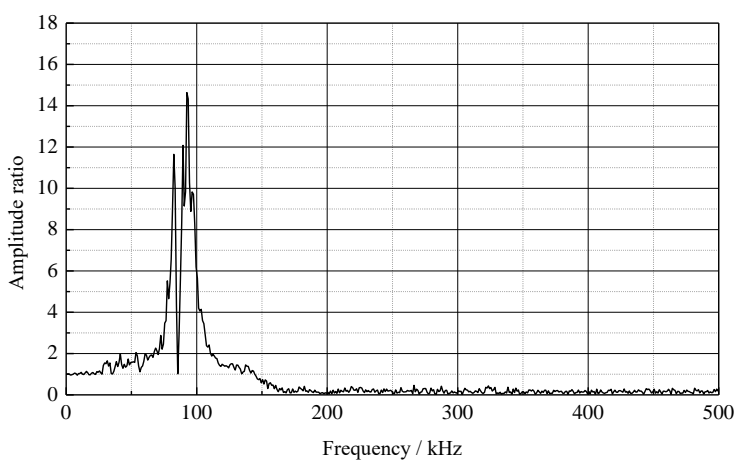

(a)

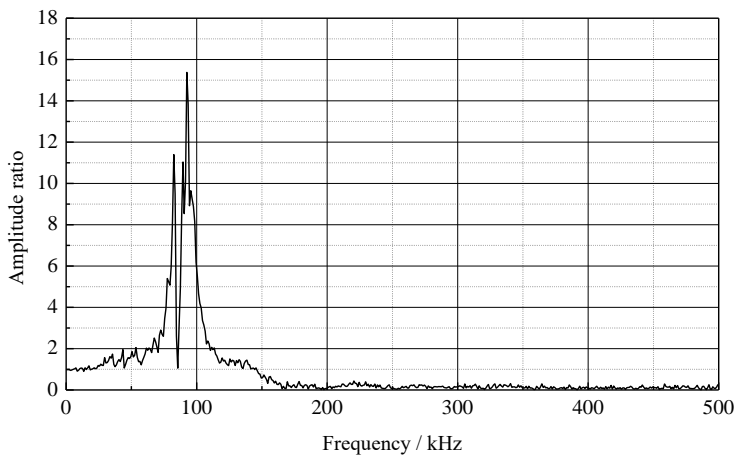

(b)

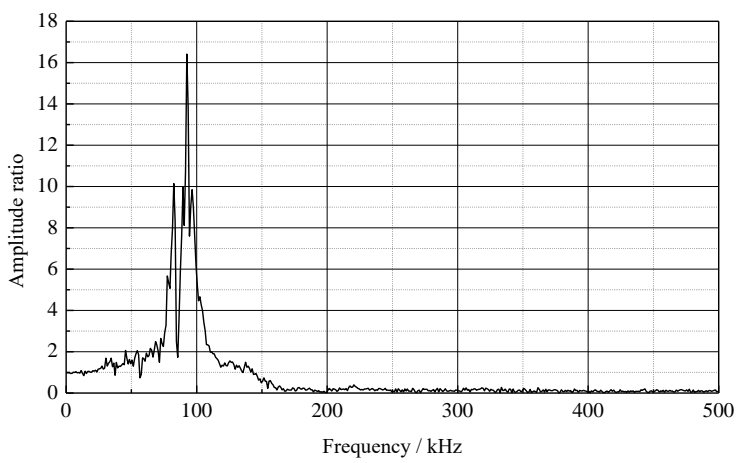

(c)

Figure 5: Amplitude-frequency characteristics obtained at: (a) $p_{4, \mathrm{~g}}=3 \mathrm{MPa}$, (b) $p_{4, \mathrm{~g}}=5 \mathrm{MPa}$, (c) $p_{4, \mathrm{~g}}=7 \mathrm{MPa}$

Figure 6 presents the phase-frequency characteristics of the piezoelectric pressure measurement system. While the characteristics are similar up to the frequency of $200 \mathrm{kHz}$, they start to differ at higher frequencies. The natural frequency of the pressure measurement system under test determined as the frequency at the phase lag $\varphi=90^{\circ}$, which was obtained with the use of a linear interpolation between the two most adjacent values of the phase lags, is approximately $81 \mathrm{kHz}$. 


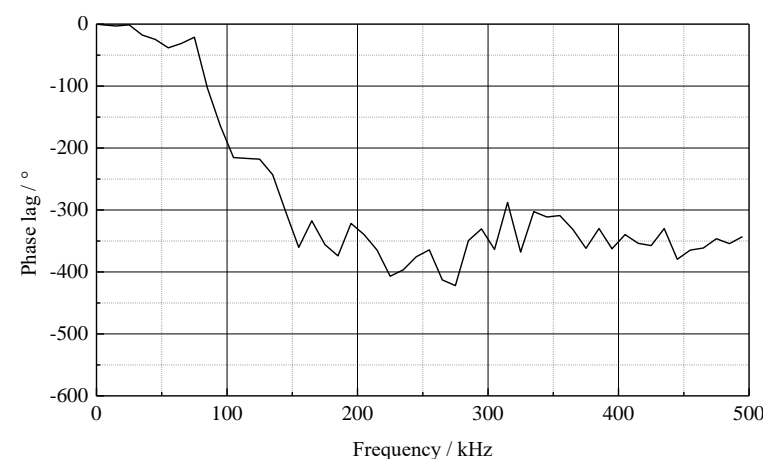

(a)

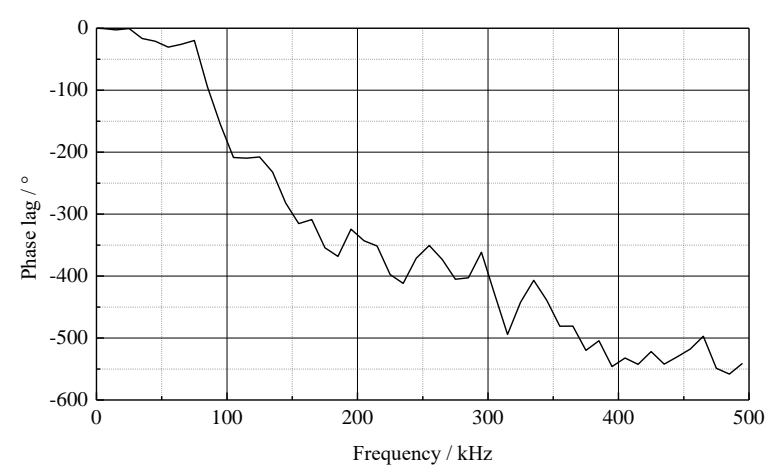

(b)

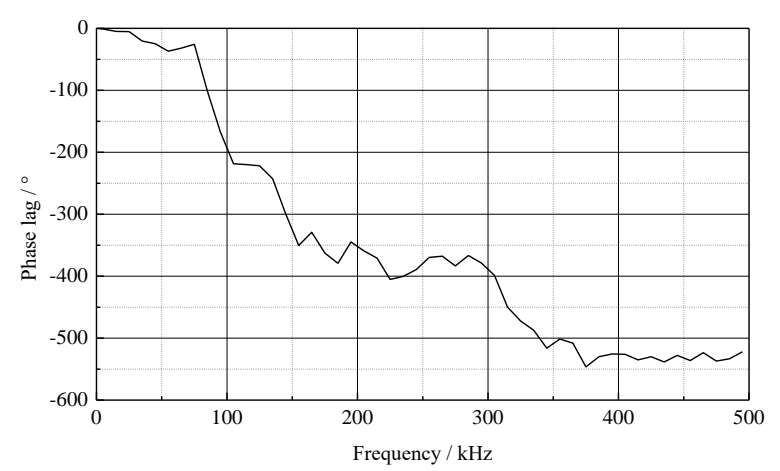

(c)

Figure 6: Phase-frequency characteristics obtained at: (a) $p_{4, \mathrm{~g}}=3 \mathrm{MPa}$, (b) $p_{4, \mathrm{~g}}=5 \mathrm{MPa}$, (c) $p_{4, \mathrm{~g}}=7 \mathrm{MPa}$

\section{SUMMARY}

The developed diphragmless shock tube has a capability to act as a primary time-varying pressure calibration standard. In the future, the shock tube will be improved by employing additional side-wall pressure transducer near the end of the tube in order to enable to describe nonlinear decelerations of the normal shock waves along the tube and therefore better predict the generated pressure steps and the arrival times of the shock front at the end-wall. Better accuracy of the obtained characteristics of the pressure measurement systems under test could be further improved also by acquiring longer post-step signals with higher sampling frequency.

\section{REFERENCES}

[1] J. Hjelmgren, Dynamic Measurement of Pressure A Literature Survey. Borås: SP Swedish National Testing and Research Institute, 2002.

[2] D. W. Holder, D. L. Schultz, On the Flow in a Reflected-Shock Tunnel. London: Her Majesty's Stationery Office, 1962.

[3] H. J. Pain, E. W. E. Rogers, "Shock Waves in Gases", Reports on Progress in Physics, vol. 25, no. 1, pp. 287-336, January 1962.

[4] J. N. de Souza Vianna, A. B. de Souza Oliveira, J. P. Damion, "Influence of the Diaphragm on the Metrological Characteristics of a Shock Tube", Metrologia, vol. 36, no. 6, pp. 599-603, December 1999.

[5] W. L. Briggs, V. E. Henson, The DFT: An Owner's Manual for the Discrete Fourier Transform. Philadelphia: Society for Industrial and Applied Mathematics, 1995.

[6] W. L. Gans, N. S. Nahman, "Continuous and Discrete Fourier Transforms of Steplike Waveforms", IEEE Transactions on Instrumentation and Measurement, vol. IM-31, no. 2, pp. 97-101, June 1982.

[7] A. Svete, J. Kutin, "Characterization of a Newly Developed Diaphragmless Shock Tube for the Primary Dynamic Calibration of Pressure Meters", Metrologia, accepted manuscript, 2020. 